ratio was significantly higher in the controls (empty gap $=$ normal healing; $n=8$ per timepoint) as compared to the Lyostypt ${ }^{\circledR}$ group ( $n=8$ per timepoint) 2 weeks after osteotomy and slightly higher after 3 weeks. Histological investigation showed the clear presence of the scaffold on both timepoints without briding cartilaginous tissue. The cell number as well as the vessel formation was significantly reduced within the fracture gap.

Conclusions: The results obtained so far support the hypothesis that we were able to develop a delayed healing or even non-union osteotomy model in mice by avoiding to create a critical size defect. Therefore, this approach represents a promising alternative animal model to evaluate therapeutic strategies to overcome bone healing complications in RA patients and perhaps other immunologically restricted patients.

References:

[1] Hoff et al. 2011 Immunol Res 51(1):116-22.

Disclosure of Interest: None declared

DOI: 10.1136/annrheumdis-2017-eular.6578

\section{AB0061 35SULPHATE INCORPORATION ASSAY AS A NEW TOOL FOR MEASURING EARLY CARTILAGE DEGRADATION FOLLOWING BLOOD EXPOSURE IN VITRO AND IN VIVO IN F8 KO RATS}

A.E. Pulles ${ }^{1,2}$, K.R. Christensen ${ }^{3,4}$, K. Coeleveld ${ }^{1}$, M. Kjelgaard-Hansen ${ }^{4}$, R.E. Schutgens ${ }^{2}$, F.P. Lafeber ${ }^{1}$, K. Roepstorff ${ }^{4}$, S.C. Mastbergen ${ }^{1} .{ }^{1}$ Department of Rheumatology \& Clinical Immunology; ${ }^{2}$ Van Creveldkliniek, University Medical Center Utrecht, Utrecht, Netherlands; ${ }^{3}$ Veterinary Disease Biology, University of Copenhagen, Copenhagen; ${ }^{4}$ Global research, Novo Nordisk A/S, Maaloev, Denmark

Background: Joint damage upon bleeding causes significant morbidity in patients with hemophilia, and adds to joint degeneration after trauma or major joint surgery. To study the pathophysiology, rodent models with histology as primary outcome measure are often used. However, histological changes take time to develop and are subject to interpretation. Determining proteoglycan synthesis rate (PSR) in cartilage, by incorporation of radioactive ${ }^{35}$ sulphate, is a sensitive method previously applied in human tissue and larger animal models to detect early cartilage changes. Isolating cartilage of small animals can be challenging, so a technique to shave off rat cartilage was developed.

Objectives: To study cartilage degradation following blood exposure, applying the ${ }^{35}$ sulphate incorporation assay on rat tibial cartilage.

Methods: A total of 13 factor VIII knock out (F8KO) rats were bred and housed at Novo Nordisk A/S, Maaloev, Denmark. After euthanasia, the legs were removed and transported to UMC Utrecht, The Netherlands.

In vitro: within 24 hours after euthanasia the cartilage of 6 healthy F8KO rats was obtained by shaving off cartilage pieces of the tibia plateau by use of a scalpel. All cartilage explants were cultured for four days; in addition to culture medium, half of the cartilage samples were cultured with $50 \% \mathrm{v} / \mathrm{v}$ whole blood. After four days PSR was determined by adding $4 \mathrm{uCi} \mathrm{Na}_{2}{ }^{35} \mathrm{SO}_{4}$ to the cultures for four hours. ${ }^{35} \mathrm{SO}_{4}^{2-}$ is incorporated into newly synthesized proteoglycans. After digesting the cartilage pieces and precipitating the proteoglycans with cetylpyridinium chloride, the amount of radioactivity was measured by liquid scintillation analysis and normalized to the specific activity of the pulse medium, labeling time and wet cartilage weight.

In vivo: in 7 F8KO rats a unilateral joint bleed was induced by needle puncture and in the following four days until euthanasia, the animals received analgesia. At UMCU, the tibial cartilage was removed and PSR determined as described above.

All animal experiments were performed in accordance with and approved by the Danish Animal Experiments Council, Ministry of Food, Agriculture and Fisheries, Denmark.

Results: On average, a total of $1.6 \mathrm{mg}(0.8-3.1 \mathrm{mg})$ cartilage per tibia could be obtained. The PSR of healthy cartilage determined after four days of culturing in vitro was on average $49.5 \mathrm{nmol} / \mathrm{h} . \mathrm{g}$. In vitro blood exposure resulted in a diminished synthesis: $7.7 \mathrm{nmol} / \mathrm{h} . \mathrm{g}(\mathrm{p}=0.0191)$, corresponding to an $84 \%$ decrease comparable to previously published experiments using human tissue [1]. In vivo, an induced joint bleed led to a $44 \%$ decrease in PSR (13.5 vs 7.5 nmol/h.g, $\mathrm{p}=0.0151$ ).
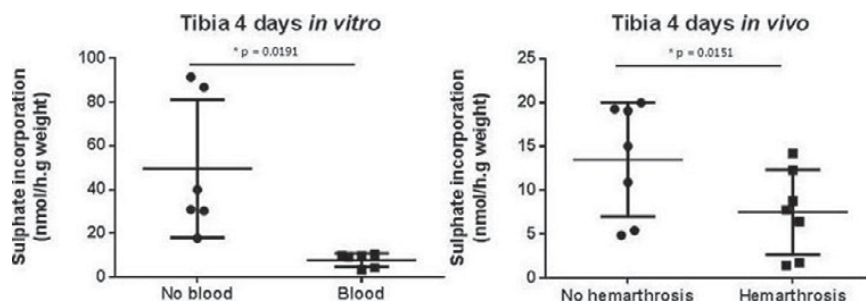

Conclusions: This study demonstrates for the first time that PSR, by use of the ${ }^{35}$ sulphate incorporation assay, can be determined in rat tibial cartilage and is affected by blood exposure in vitro and in vivo. As such, this assay can be a valuable tool to detect cartilage changes using joint degenerative rat models. References:

[1] L. van Vulpen, R. Schutgens, K. Coeleveld, E. Alsema, G. Roosendaal,
S. Mastbergen, F. Lafeber. IL-1beta: in contrast to TNFalpha, is pivotal in blood-induced cartilage damage and is a potential target for therapy. Blood, 2015;126(19):2015, pp.2239-2246.

Disclosure of Interest: None declared

DOI: 10.1136/annrheumdis-2017-eular.1468

\section{AB0062 EX VIVO BACK-TRANSLATION OF FOSTAMATINIB'S EFFECT ON JOINT ECM TURNOVER SHOWS SIGNIFICANT EFFECT ON BONE BUT NO EFFECT ON THE SYNOVIUM}

C.F. Kjelgaard-Petersen ${ }^{1,2}$, T.G. Christensen ${ }^{3}$, P. Hägglund ${ }^{2}$, M.A. Karsdal ${ }^{4}$ C.S. Thudium ${ }^{4}$, A.-C. Bay-Jensen ${ }^{4} .{ }^{1}$ Biomarkers and Reseach, Rheumatology, Nordic Bioscience, Herlev; ${ }^{2}$ Bioengineering. Technical University of Denmark, Lyngby: ${ }^{3}$ Orthopaedic surgery Unit, Gentofte University Hospital, Gentofte;

${ }^{4}$ Biomarkers and Research, Rheumatology, Nordic Bioscience, Herlev, Denmark

Background: It is important to establish translational methods that aid in pre-clinical go/no-go decision points to increase the success rate of approved DMARDs. The Spleen tyrosine kinase (Syk) inhibitor Fostamatinib (Fosta) was terminated for development for RA, due to insufficient effect on joint structure in phase III.

Objectives: The objective was to use a translational system of ex vivo cultures to back-translate the insufficient effect on joint structure described in clinical studies. Methods: Human mature osteoclasts (HOC) seeded on bone, bovine cartilage explants (BEX) and human synovial explants (SME) were treated with R406 (API of Fosta) at $5 \mu \mathrm{M}-0.05 \mu \mathrm{M}$. Osteoclasts were co-stimulated with $25 \mathrm{ng} / \mathrm{ml}$ M-CSF and RANKL, while BEX and SME were co-stimulated with TNF $\alpha 2 \mathrm{ng} / \mathrm{mL}$ and OSM $10 \mathrm{ng} / \mathrm{mL}(\mathrm{O}+\mathrm{T})$ or TNF $\alpha 10 \mathrm{ng} / \mathrm{mL}$, respectively. CTX-1 and $\mathrm{Ca}^{2+}$ were measured in conditioned medium (CM) from HOC. Metabolic activity of $\mathrm{HOC}$ was assessed with Alamar Blue. C2M and AGNx1 were measured in CM from BEX, while acMMP3, C1M, and C3M were measured in CM from SME. The biomarkers in BEX and SME CM were measured at 4 time points and the total release were quantified by area under the curve (AUC). CTX-1, C2M, AGNx1, acMMP3, $\mathrm{C} 1 \mathrm{M}, \mathrm{C} 2 \mathrm{M}$ and $\mathrm{C} 3 \mathrm{M}$ were measured with ELISA. $\mathrm{Ca}^{2+}$ was measured with ADVIA Chemistry system. Statistical differences of metabolic activity was calculated with One-way ANOVA with Dunnett's multiple comparison test. Statistical differences between biomarkers levels or AUC were calculated with Kruskall Wallis test with Dunn's multiple comparision test.

Results: R406 decreased the release of CTX-1 (Fig 1A) and $\mathrm{Ca}^{2+}$ in a dosedependent manner, with a significant decrease at $1 \mu \mathrm{M}(P<0.01)$. This might be due to a toxic effect of R406 on HOC (Fig 1B) $(P<0.05)$. R406 decreased the total release of C2M and AGNx1 in a dose-dependent manner in BEX. C2M was inhibited a concentrations down to $1.25 \mu \mathrm{M}(P=0.034)$, and $A G N \times 1$ down to $5 \mu \mathrm{M}$ $(P=0.012)$. In SME R406 only decreased the release of C1M and C3M (Fig 1e) significantly at $5 \mu \mathrm{M}(\mathrm{C} 1 \mathrm{M}: P=0.03$, C3M: $P=0.046)$, and tended to decrease release of acMMP3 (Fig 1f) at $5 \mu \mathrm{M}$. The later was however not significant.

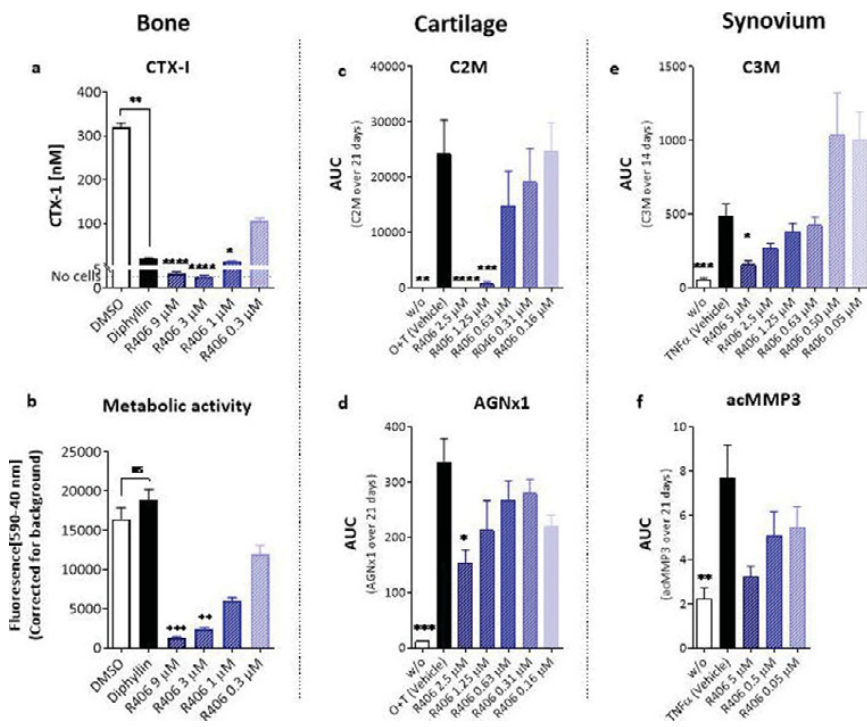

Conclusions: Serum-based biomarkers of the joint ECM turnover were measured in CM from HOC, cartilage and synovial explant cultures. R406 decreased bone resorption and $\mathrm{HOC}$ metabolic activity in a dose-dependent manner, together with the MMP-mediated degradation of type II (C2M) collagen and aggrecanse degradation of aggrecan (AGNx1) in cartilage. However, R406 had limited effect on the inflammation driven MMP-mediated degradation of type I (C1M) and III (C3M) collagen and activation of MMP-3. CTX-1, C2M, and C3M have previously been measured in OSKIRA-1, with a profile identical to the ex vivo measurements here [1].

References:

[1] Platt A, Bay-Jensen AC, Braddock M, et al. The Effect of Fostamatinib with 